\title{
The REBECGA Brazilian Registry of Pregnancy and Heart Disease: Rationale and Design
}

Walkiria Samuel Avila, ${ }^{\oplus}$ Maria Alayde Mendonça Rivera, ${ }^{2,3}$ Celi Marques-Santos, ${ }^{3 \oplus}$ Ivan Romero Rivera, ${ }^{2 \oplus}$ Maria Elizabeth Navegantes Caetano Costa ${ }^{4 \oplus}$ Alexandre Jorge Gomes de Lucena, ${ }^{5 \oplus}$ Claudia Maria Vilas Freire, ${ }^{6 \oplus}$ Regina Coeli Marques de Carvalho, ${ }^{7,-}$ Daniel Born, ${ }^{9 \oplus}$ Felipe Favorette Campanharo, ${ }^{9 \oplus}$ Fabio Bruno Silva ${ }^{10}$

Instituto do Coração da Faculdade de Medicina da Universidade de São Paulo (InCor-FMUSP), ' São Paulo, SP - Brazil

Hospital Universitário da Universidade Federal de Alagoas, ${ }^{2}$ Maceió, AL - Brazil

Santa Casa de Misericordia de Maceió, ${ }^{3}$ Maceió, AL - Brazil

Universidade Tiradentes, ${ }^{3}$ Aracaju, SE - Brazil

Fundação Pública Estadual Hospital de Clínicas Gaspar Viana, ${ }^{4}$ Belém, PA - Brazil

Hospital Agamenon Magalhães, ${ }^{5}$ Recife, $P E-$ Brazil

Hospital das Clinicas da Universidade Federal de Minas Gerais, ${ }^{6}$ Belo Horizonte, MG - Brazil

Hospital Geral de Fortaleza, Fortaleza, CE - Brazil

Secretaria de Saúde do Estado do Ceará (SESA), ${ }^{8}$ Fortaleza, CE - Brazil

Hospital São Paulo - Universidade Federal de São Paulo, ${ }^{9}$ São Paulo, SP - Brazil

Instituto Dante Pazzanese de Cardiologia, ${ }^{10}$ São Paulo, SP-Brazil

\section{Abstract}

Background: Maternal mortality rates in Brazil remain above the goals established by the United Nations Sustainable Development Goals. Heart disease is estimated to affect $4 \%$ of all pregnancies and remains by itself the main indirect obstetric cause of maternal death. In the last decades, a significant improvement in the prognosis of heart diseases has made pregnancy possible in women with heart disease and provided better maternal and fetal outcomes.

Objectives: To establish a multicenter Brazilian Registry of pregnant women with heart disease; to study the causes of immediate and late maternal mortality; and to assess the prevalence of heart disease in the country's macro-regions.

Methods: This is an observational study, with retrospective and prospective stages, of the clinical and obstetric progression of pregnant women with heart disease. These women consecutively received care during pregnancy and will be followed up for up to a year after delivery at public and private hospitals with infrastructure for the execution of this project, a principal investigator, and approval by Ethics and Research Committees.

Results: Our results will be presented after data collection and statistical analysis, aiming to demonstrate immediate and late maternal mortality rates, as well as the prevalence of heart disease in the country and its cardiovascular and obstetric complications during pregnancy.

Conclusions: REBECGA will be the Brazilian Registry of heart disease and pregnancy and it will contribute to planning preventive measures, raising financial resources for the improvement of high-risk prenatal care, and reducing immediate and late maternal mortality due to heart disease.

Keywords: Pregnancy; Heart Diseases; Maternal Mortality; Cardiovascular Pregnancy Complications.

\section{Introduction}

Maternal mortality is a severe social problem in Brazil, reflecting our condition as an emerging country. Assistance to high-risk pregnant women displays

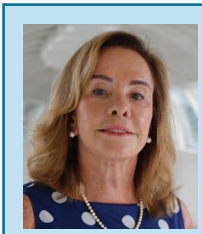

Walkiria Samuel Avila, MD, PhD

Coordinator of the Heart Disease and Pregnancy and Reproductive Counseling Program at InCorHCFMUSP

Hospital das Clínicas, Faculty of Medicine, University of São Paulo

Mailing Address: Maria Alayde Rivera

Av. Lourival Melo Mota, s/n. Postal Code: 57072-900, Tabuleiro do Martins, Maceió, AL - Brazil.

E-mail: malayde1@uol.com.br 
profound differences, specifically considering those with heart disease and the proportion of avoidable deaths, in the various regions of Brazil. ${ }^{1}$

In the last 3 decades, Brazil reported a marked reduction in maternal mortality rate - from 143.2 to 64 deaths per 100000 live births. However, these coefficients are higher than those established by the United Nations Sustainable Development Goals and 6 times higher than those accepted by the World Health Organization. ${ }^{2}$

Heart disease is the main indirect obstetric cause of maternal death, being present in $4 \%$ of pregnancies. The impact of heart disease in maternal mortality was investigated in 27 referral obstetric units, and maternal death showed a prevalence ratio that was 4 times higher (4.8 vs 1.2 ) in pregnant women with heart disease. ${ }^{3}$

In Brazil, rheumatic disease contributes to an expressive incidence of acquired heart disease in young women, with a 4 times higher prevalence ratio when compared to developed countries. The growing population of pregnant women with congenital heart disease and cardiomyopathies has also contributed to significant complication and maternal mortality rates during pregnancy. ${ }^{4}$

In the last decades, a significant improvement in the prognosis of cardiovascular diseases and a higher life expectancy for children and adolescents with heart disease has been observed, including the safe development of pregnancy. However, the country lacks national data on these changes.

Worldwide multicenter registries, guidelines, and positions have been established with the commitment of reducing maternal mortality due to heart disease..$^{5-9}$ In clinical practice, the applicability of maternal cardiovascular risk scores published in the international literature faces serious limitations, especially because the epidemiology of heart diseases in reproductive age and the social and demographic characteristics of the Brazilian population are very diverse.

In agreement with the current global movement, the elaboration of the Brazilian Registry of Pregnancy and Heart Diseases, led by the Women's Cardiology Department (DCM) and hereinafter named the REBECGA Registry, responds to our social responsibility regarding the quality of life of women with heart disease. Notably, it contributes to planning preventive measures, raising financial resources for providing better prenatal care, and reducing immediate and late maternal mortality due to heart diseases manifested during pregnancy.
The primary objective of the REBECGA Registry is the construction of a national register of heart diseases manifested during pregnancy, focusing on the study of immediate and late maternal mortality due to heart disease. Our secondary objectives are the identification of the cardiovascular complications of pregnancy and the prevalence of these heart diseases in the country's macro-regions.

\section{Methods}

This is an observational study with a retrospective (cross-sectional) stage and a prospective (longitudinal) stage, including women with heart disease who consecutively received care during pregnancy and were followed up for up to a year after delivery. We will analyze pre-selected maternal, obstetric, and fetal variables in both stages of the registry (Chart 1). Data from the retrospective stage, which ranged from 2017 to 2020, will be obtained through the analysis of medical records and or/telephone contact; as for the prospective stage (from 2021 to 2026), they will be collected at inperson periodical visits through paper forms and further storage on an online platform. During the prospective stage, clinical and obstetric approaches should follow the recommendations by the Brazilian Society of Cardiology (SBC) Statement for Management of Pregnancy and Family Planning in Women with Heart Disease - 2020 in order to reduce discrepancies in results.

All participants of the registry or their legal representatives must sign the free and informed consent form (FICF). In case the patient has not reached legal age, the FICF shall be signed by her legal guardian. Patients who do not attend scheduled visits after delivery will be interviewed by telephone for collecting obstetric and perinatal information. We have not anticipated complementary examinations. The participant hospitals, whether public or private, should present: 1) approval of the project by the Research and Ethics Committee of the participating institution; 2) infrastructure for executing the project; 3) a principal investigator who will be responsible for the Registry until the end of the project.

Inclusion and exclusion criteria: We will include pregnant women with heart disease, with anatomical and etiological diagnosis defined at the first prenatal consult, regardless of their involvement in other clinical studies, after signing of the FICF. Structural and electrical heart diseases, as well as complicating factors included in the Registry platform, are presented on Charts 2 and 3, respectively. 


\section{Chart 1 - Variables included in the registry}

Patient identification: record number, age, date of birth, gestational age at first consult;

Characterization of heart disease: anatomical and etiological diagnosis, previous cardiovascular intervention (type and time/years), complicating factors, associated structural heart injury;

Obstetric history: number of pregnancies, deliveries, and miscarriages;

Clinical characteristics: New York Heart Association (NYHA) functional classification at first prenatal consult;

Comorbidities: chronic arterial hypertension, bronchial asthma, gestational diabetes, thyroid disorders, obesity (body mass index [BMI] $>30$ $\left.\mathrm{Kg} / \mathrm{m}^{2}\right)$;

Electrocardiographic variables (12-lead recording);

Echocardiographic variables (two-dimensional transthoracic echocardiography)

Cardiac biomarkers: natriuretic peptide (BNP), troponin, international normalized ratio (INR), anti-factor Xa, others;

Cardiovascular complications during pregnancy;

Pharmacological intervention

Anticoagulation - Use in the first, second, and third trimester, as well as on the seventh, 14th, and 42nd day after delivery. Anticoagulants considered for the registry: low molecular weight heparin, unfractionated heparin, warfarin;

Non-pharmacological intervention, considering percutaneous intervention or heart surgery performed during pregnancy or puerperium;

Type and date of delivery, gestational age at delivery (in weeks);

Type of anesthesia used during delivery;

Neonatal complications related to maternal heart disease (congenital malformations or consequences of medications used during pregnancy);

Cardiovascular complications during delivery and puerperium

Obstetric complications of pregnancy, delivery, and puerperium;

Hospitalization for treating cardiovascular complications during pregnancy or puerperium;

Late maternal death: considering 12 months after delivery.

\section{Chart 2 - Heart disease diagnosis}

\begin{tabular}{lc}
\hline Valvular heart disease & Congenital heart disease \\
\hline Cardiomyopathies & Pericardial disease \\
\hline Aortic diseases & Ischemic heart disease \\
\hline Inflammatory or infiltrative heart disease & \\
\hline Cardiac arrhythmia with intervention indication & \\
\hline
\end{tabular}

\section{Chart 3 - Complicating factors}

Ventricular dysfunction

Pulmonary hypertension

Residual injury in the late postoperative period

Graft or prosthesis dysfunction

\section{Fibrillation/atrial flutter}

Hypoxemia 
Chart 4-Maternal cardiac complications

\begin{tabular}{lc}
\hline Heart failure & Acute pulmonary edema \\
\hline Cardiac syncope & Acute myocardial infarction \\
\hline Aortic dissection & Infective endocarditis \\
\hline Arterial or venous thromboembolism & \\
\hline Need for anticoagulants & \\
\hline Cardiorespiratory arrest & \\
\hline Non-pharmacological intervention & \\
\hline
\end{tabular}

Chart 5 - Pharmacological treatment during pregnancy

\begin{tabular}{lc}
\hline Loop diuretics & Beta-blockers \\
\hline Calcium channel blockers & Antiarrhythmics \\
\hline Vasodilators & Anticoagulants \\
\hline Platelet antiaggregants & Others \\
\hline
\end{tabular}

Women to be excluded from the study are 1) patients with severe diseases (renal, hepatic, lymphatic, neoplasms, and others); 2) patients with conditions that interfere in their participation or capacity of concluding the study; 3) those who did not present structural or electrical heart disease. The studied variables will include clinical, obstetric, and fetal complications, as well as pharmacological and interventional management during pregnancy (Charts 4 and 5).

Results of the 12-lead electrocardiography and the two-dimensional transthoracic echocardiography will be analyzed, and echocardiographic results related to the structural cardiac injury will be considered. For example, the echocardiographic data obtained for valvular heart disease will not be the same as those for congenital heart disease or cardiomyopathies, and so on. Cardiac biomarkers will only be considered in case of acute cardiac events occurring during pregnancy.

\section{Data recording}

Variables selected in this study (Charts 1-6) for each patient will be stored in the Research Electronic Data Capture (REDCap) platform, individually and consecutively, in a specific form dedicated to this registry (Table 1). Professionals responsible for collecting and storing data in the various participant centers will undergo initial online training for guaranteeing quality in data collection. The principal investigator in each center will be responsible for consecutively recording data obtained on the selected patients.

\section{Expected outcomes}

Primary: immediate and late mortality rates due to heart disease;

Secondary: cardiac, obstetric, and fetal complications and need for pharmacological or non-pharmacological intervention related to heart diseases.

\section{Data management and quality assurance}

The REDCap platform was chosen because Hospital das Clínicas da Faculdade de Medicina da Universidade de São Paulo (HC-FMUSP) has the software license and thus no additional costs with data management would be generated for the project. In each participating center, the team will fill out an electronic form at the REDCap platform. Data management in the REBECGA registry will include the following steps: 1) a REDCap coordinating team will undergo training sessions before the beginning of the study for guaranteeing consistency throughout the project's procedures, including data collection and report generation; 2) participating institutions will receive an Operations Manual with the 


\begin{tabular}{|c|c|}
\hline 1) & Patient identification: record number, age, date of birth, gestational age at first consult; \\
\hline 2) & $\begin{array}{l}\text { Characterization of heart disease: anatomical and etiological diagnosis, previous cardiovascular intervention (type and time/years), } \\
\text { complicating factors, associated structural heart injury; }\end{array}$ \\
\hline 3) & Obstetric history: number of pregnancies, deliveries, and miscarriages; \\
\hline 4) & Clinical characteristics: New York Heart Association (NYHA) functional classification at first prenatal consult; \\
\hline 5) & $\begin{array}{l}\text { Comorbidities: chronic arterial hypertension, bronchial asthma, gestational diabetes, thyroid disorders, obesity (body mass index } \\
\left.[\mathrm{BMI}]>30 \mathrm{Kg} / \mathrm{m}^{2}\right)\end{array}$ \\
\hline 6) & Electrocardiographic variables; \\
\hline 7) & Echocardiographic variables (two-dimensional transthoracic echocardiography) \\
\hline 8) & Cardiac biomarkers: natriuretic peptide (BNP), troponin, international normalized ratio (INR), anti-factor Xa, others; \\
\hline 9) & Cardiovascular complications during pregnancy; \\
\hline 10) & Pharmacological intervention considering the class of medications with cardiovascular action; \\
\hline 11) & $\begin{array}{l}\text { Anticoagulation - Use in the first, second, and third trimester, as well as on the seventh, } 14 \text { th, and 42nd day after delivery. } \\
\text { Anticoagulants considered for the registry: low molecular weight heparin, unfractionated heparin, warfarin; }\end{array}$ \\
\hline 12) & $\begin{array}{l}\text { Non-pharmacological intervention, considering percutaneous intervention } \\
\text { or heart surgery performed during pregnancy or puerperium; }\end{array}$ \\
\hline 13) & Type and date of delivery, gestational age at delivery (in weeks); \\
\hline 14) & Type of anesthesia used during delivery; \\
\hline 15) & $\begin{array}{l}\text { Neonatal complications related to maternal heart disease } \\
\text { (congenital malformations or consequences of medications used during pregnancy); }\end{array}$ \\
\hline 16) & Cardiovascular complications during delivery and puerperium; \\
\hline 17) & Obstetric complications of pregnancy, delivery, and puerperium; \\
\hline 18) & Hospitalization for treating cardiovascular complications during pregnancy or puerperium; \\
\hline 19) & Late maternal death: considering time after delivery in months, cardiac and obstetric causes, or others. \\
\hline
\end{tabular}

description of each step of the protocol and will have telephonic support for solving problems or doubts; 3) the Academic Research Organization (ARO) of the project's coordinating center will analyze monthly reports, rates of events, and cardiac outcomes; 4) the database centralized at the coordinating center will perform internal verifications of validity and variation for identifying errors or omissions and notifying the participating institutions; 5) in the prospective stage, we will accept a loss to follow-up of up to $5 \%$; 6) the coordinating team will perform an inspection at institutions with monitoring purposes, if necessary.

\section{Project management}

The study will have an investigating committee constituted by members of the DCM of the SBC, being responsible for selecting participating centers and managing the data registry platform. In each center, the principal investigator is committed to selecting patients, managing incomplete data, storing data on the REDCap platform, and participating in periodical meetings with the management committee for decisionmaking regarding the progress of the study.

\section{Sample size}

We will perform 3 intermediate analyses throughout the study: 1) the retrospective stage, corresponding to the period between 2017 and $2020 ; 2)$ the prospective stage - phase 1 , which corresponds to the period between 2021 and 2023; and 3) the prospective stage - final phase, between 2024 and 2026. In the retrospective stage, we will use convenience sampling including pregnant women who consecutively received care between 2017 and 2020. In the prospective stage, our sample calculation estimates 300 to 350 women/year, which corresponds to a mean number of 40 to 70 women/year to receive care at the participating institutions. 


\section{Statistical analysis}

The normality of data distributions will be verified through a Shapiro-Wilk test, with a significance level of $5 \%$, in addition to a graphical analysis by gg-plot and histograms. Continuous variables with normal distribution will be presented as means and standard deviations, while those with non-normal distributions will be presented as absolute and relative frequencies, with the respective confidence intervals. For determining the association between categorical variables, we will use the chi-squared test where the odds ratio will be established for dependent variables with a significance level of $5 \%$ and a $95 \%$ confidence interval. For quantitative variables, Pearson's correlation coefficient will be employed for determining the correlation between variables, based on the same significance level. Estimates for quantitative variables with normal distribution will use the unpaired Student's t-test with a 5\% significance level. Non-parametric variables, if dependent, will be compared via a Wilcoxon test; if independent, the Mann-Whitney test will be employed for statistical inference. All analyses will be performed using R software, version 4.0.5.

\section{Ethical aspects}

This study was approved by the Ethics and Research Committee of HC-FMUSP - Protocol No. SDC5232/21/007 - and will be conducted according to the current Brazilian legislation on research ethics, including Resolutions 466/2012-CNS-CONEP and 510/2016-CNS. This clearance allows each participating center to submit a protocol to local committees. Eventual amendments to the protocol will also require approval by the ethics committees, if applicable, according to local laws and regulations.

Confidentiality regarding the study data will be guaranteed by the safe electronic retransmission to the coordinating center, with strictly confidential data storage and maintenance according to the current legislation. Participants will not be identified at any time.

The study will be conducted by the Clinical Research Projects Committee of the DCM of the SBC, and its content is intellectual property of this committee; the use of the study data for other ends that not the REBECGA Registry is thus considered illegal.

\section{Result publication}

Publications related directly to the main results of the REBECGA Registry will be produced with group authorship and the roles of all investigators will be recognized in an appendix and under the responsibility of the Registry's Investigating Committee. Subsequent publications will be authored by specific individuals, representing investigators of the REBECGA Registry. The selection of professionals for leading the writing of these subsequent publications by the Registry's Investigating Committee will depend on their roles and contributions to the study, as well as on their interests and scientific knowledge. Data will be published after conclusion of the 3 stages and will be available in the DCM at the SBC website.

Coordinating center: Project Management Unit of Instituto do Coração (InCor), HC-FMUSP, Rua Dr Enéas Carvalho de Aguiar, No. 44, Cerqueira César -SP. Postal Code: 05403-000.

\section{Funding}

The REBECGA registry is property of the DCM in partnership with the SBC and will use financial resources allocated to its execution. Resources will be raised with the support of the SBC and Research Support Foundations (FAPs) of each state involved in the study. The project will be submitted to PROADS, CNPq and CAPES after their respective calls for proposals.

\section{Study limitations}

In the retrospective stage, data loss could happen when considering that no uniform protocol was adopted beforehand. In the prospective stage, there will be no control group since this is an observational study. Resource inequalities, the fact that the maternity ward is located near the cardiology center, and differences in the number of patients in each center will be considered in the statistical analysis. At this moment, the South region does not have a representing institution fulfilling the criteria established by our methodology.

\section{Acknowledgments}

Iris Tikkanen Belitsky, graduate student in Bioinformatics at the Inter-unit Graduate Program in Bioinformatics of Universidade de São Paulo (IME-USP), for her consultancy in our statistical analysis.

Member of the Investigating Committee and president of the DCM (biennium 2020-2021) of the SBC Dr. Celi Marques Santos. 
Participating institutions - principal investigators

InCor-FMUSP - Prof. Dr. Walkiria Samuel Avila; Hospital Geral de Fortaleza - Secretaria de Saúde do Estado do Ceará (SESA) - Dr. Regina Coeli Marques de Carvalho; Hospital das Clínicas da Universidade Federal de Minas Gerais - Dr. Claudia Maria Vilas Freire; Instituto Dante Pazzanese de Cardiologia - Dr. Fabio Bruno Silva; Hospital Universitário da Universidade Federal de Alagoas - Dr. Maria Alayde Mendonça Rivera and Dr. Ivan Romero Rivera; Hospital Agamenon Magalhães - Dr. Alexandre Jorge Gomes de Lucena; Hospital São Paulo - Universidade Federal de São Paulo - Escola Paulista de Medicina - Dr. Felipe Favorette Campanharo and Dr. Daniel Born; Fundação Pública Estadual Hospital de Clínicas Gaspar Viana - Secretaria de Saúde do Estado do Pará; Dr. Elizabeth Caetano.

\section{Author contributions}

Conception and design of the research: Avila WS, Rivera MAM, Marques-Santos C, Rivera IR, Costa MENC, Lucena AJG, Freire CMV, Carvalho RCM, Born D, Campanharo FF, Silva FB. Acquisition of data: Avila WS, Rivera MAM, Marques-Santos C, Rivera IR, Costa MENC, Lucena AJG, Freire CMV, Carvalho RCM, Born D, Campanharo FF, Silva FB. Analysis and interpretation of the data: Avila WS, Rivera MAM, Marques-Santos C, Rivera IR, Costa MENC, Lucena AJG, Freire CMV, Carvalho RCM, Born D, Campanharo FF, Silva FB. Statistical analysis: Avila WS, Rivera MAM, Marques-Santos C, Rivera IR, Costa MENC, Lucena AJG, Freire CMV, Carvalho RCM, Born D, Campanharo FF, Silva FB. Obtaining financing: Avila WS, Rivera MAM,

\section{References}

1. Carvalho PI, Frias PG, Lemos MLC, Frutuoso LALM, Figueirôa BQ, Pereira CCB, et al. Sociodemographic and Health Care profile of Maternal Death in Recife, PE, Brazil, 2006-2017: A Descriptive Study. Epidemiol Serv Saude. 2020;29(1):e2019185. doi: 10.5123/S1679-49742020000100005.

2. Brasil. Ministério da Saúde. Secretaria de Vigilância em Sáude. Boletim Epidemiológico;2020;51(51):1-33

3. Campanharo FF, Cecatti JG, Haddad SM, Parpinelli MA, Born D, Costa ML, et al. The Impact of Cardiac Diseases during Pregnancy on Severe Maternal Morbidity and Mortality in Brazil. PLoS One. 2015;10(12):e0144385. doi: 10.1371/journal.pone.0144385.

4. Avila WS, Rossi EG, Ramires JA, Grinberg M, Bortolotto MR, Zugaib M, et al. Pregnancy in Patients with Heart Disease: Experience with 1,000 Cases. Clin Cardiol. 2003;26(3):135-42. doi: 10.1002/clc.4960260308.

5. Silversides CK, Grewal J, Mason J, Sermer M, Kiess M, Rychel V, et al. Pregnancy Outcomes in Women with Heart Disease: The CARPREG II Study. J Am Coll Cardiol. 2018;71(21):2419-30. doi: 10.1016/j. jacc.2018.02.076.
Marques-Santos C, Rivera IR, Costa MENC, Lucena AJG, Freire CMV, Carvalho RCM, Born D, Campanharo FF, Silva FB. Writing of the manuscript: Avila WS, Rivera MAM, Marques-Santos C, Rivera IR, Costa MENC, Lucena AJG, Freire CMV, Carvalho RCM, Born D, Campanharo FF, Silva FB. Critical revision of the manuscript for intellectual content: Avila WS, Rivera MAM, Marques-Santos C, Rivera IR, Costa MENC, Lucena AJG, Freire CMV, Carvalho RCM, Born D, Campanharo FF, Silva FB.

\section{Potential Conflict of Interest}

No potential conflict of interest relevant to this article was reported.

\section{Sources of Funding}

This study was partially funded by Sociedade Brasileira de Cardiologia and Fundação de Pesquisa da USP.

\section{Study Association}

This study is not associated with any thesis or dissertation work.

\section{Ethics approval and consent to participate}

This study was approved by the Ethics Committee of the USP - Hospital das Clínicas da Faculdade de Medicina da Universidade de São Paulo - HCFMUSP under the protocol number 42739321.9.1001.0068. All the procedures in this study were in accordance with the 1975 Helsinki Declaration, updated in 2013. Informed consent was obtained from all participants included in the study.

6. Roos-Hesselink J, Baris L, Johnson M, Backer J, Otto C, Marelli A, et al Pregnancy Outcomes in Women with Cardiovascular Disease: Evolving Trends Over 10 Years in the ESC Registry of Pregnancy and Cardiac disease (ROPAC). Eur Heart J. 2019;40(47):3848-55. doi: 10.1093/eurheartj/ehz136.

7. Balci A, Sollie-Szarynska KM, van der Bijl AG, Ruys TP, Mulder BJ, Roos-Hesselink JW, et al. Prospective Validation and Assessment of Cardiovascular and Offspring Risk Models for Pregnant Women with Congenital Heart Disease. Heart. 2014;100(17):1373-81. doi: 10.1136/ heartjnl-2014-305597.

8. Pijuan-Domènech A, Galian L, Goya M, Casellas M, Merced C, FerreiraGonzalez I, et al. Cardiac Complications During Pregnancy are Better Predicted With the Modified WHO Risk Score. Int J Cardiol. 2015;195:14954. doi: 10.1016/j.ijcard.2015.05.076

9. Avila WS, Alexandre ERG, Castro ML, Lucena AJG, Marques-Santos C, Freire CMV, et al. Brazilian Cardiology Society Statement for Management of Pregnancy and Family Planning in Women with Heart Disease - 2020. Arq Bras Cardiol. 2020;114(5):849-942. doi: 10.36660/abc.20200406. 